\title{
Combination of vitamin E and L-carnitine is superior in protection against isoproterenol-induced cardiac injury: histopathological evidence
}

\author{
E.A. Huwait \\ Department of Biology, Faculty of Science, King Abdulaziz University, Jeddah, Saudi Arabia
}

[Received: 16 April 2018; Accepted: 23 May 2018]

Background: L-carnitine and vitamin E have antioxidant properties. This study aimed to assess the effectiveness of L-carnitine, vitamin $E$ and their combination in protection against isoproterenol (ISO)-induced biochemical and histopathological changes in rat heart.

Materials and methods: Fifty male Wistar rats assigned to five groups; control, ISO-treated group (100 mg/kg), ISO + vitamin E-treated group (100 IU/ $/ \mathrm{kg})$, ISO + L-carnitine $(100 \mathrm{mg} / \mathrm{kg})$ and ISO + vitamin E + L-carnitine treated group. At the end of the experiment, serum cardiac enzyme as well as the cardiac level malondialdehyde (MDA), antioxidant enzymes and inflammatory cytokines interleukin-6, tumour necrosis factor alpha (TNF- $\alpha$ ) were assessed. Histopathological changes in the left ventricle wall were assessed using the light and electron microscopy. Results: Treating rats with vitamin $E$ and L-carnitine could alleviate ISO-induced changes as it significantly reduced the serum level cardiac enzymes, MDA and IL-6, TNF- $\alpha$ and improved the antioxidants enzymes (SOD, GSPxase and GSRase). Histopathologically, they improved cardiac fibres atrophy, haemorrhages between cardiac fibres, lost striations, and disturbed sarcomere structure. The combined effect of vitamin $E$ and L-carnitine was more superior compared to the other groups. Conclusions: Combined administration of vitamin E, L-carnitine ameliorated the biochemical and histopathological cardiac injury induced by ISO. The effect seemed to be mediated through the antioxidant and anti-inflammatory effect of vitamin $E$, L-carnitine. Administration of these two elements is recommended for patient at risk for myocardial infarction. (Folia Morphol 2019; 78, 2: 274-282)

Key words: vitamin E, L-carnitine, isoproterenol, heart, histology, antioxidant, anti-inflammatory

\section{INTRODUCTION}

L-carnitine, L-trimethy I-3-hydroxy ammoniobetanoate, is a natural endogenous water soluble antioxidant located on the mitochondrial membrane and is found in all mammals. It reduces the intracellular buildup of toxic metabolites in ischaemic conditions [25]. It was reported that "Carnitine plays a pivotal role in myocardial energy metabolism through transport of long-chain fatty acyl intermediates, across the inner mitochondrial membrane for subsequent oxidation, and regulation of carbohydrate metabolism by modulation of the intramitochondrial

Address for correspondence: Dr. E.A. Huwait, Associate Professor, Department of Biology, Faculty of Science, King Abdulaziz University, Jeddah, Saudi Arabia, e-mail: dretedalahuwait@gmail.com; ehuwit@kau.edu.sa 
acetyl-CoA:CoA ratio" [26]. As such, it is used in the prevention and treatment of oxidative stress and related health problems [1]. It exerts its antioxidant potential by reducing reactive oxygen species (ROS) production and scavenging free radicals [17].

A link was reported between consumption of carnitine and cardiovascular diseases [27]. However, contradictory data were found regarding beneficial effects of L-carnitine. Liepinsh et al. [18] reported that long-term decrease in L-carnitine is important for the energy metabolism regulation and treatment of atherosclerosis and heart diseases. On the other hand, Zambrano et al. [35] reported that arterial hypertension-related cardiac fibrosis could be inhibited by L-carnitine through modulation of peroxisome proliferator activated receptor- $\gamma$ expression.

Vitamin E, a fat soluble vitamin, possesses a powerful antioxidant activity [2]. Administration of vitamin $\mathrm{E}$ was found to decrease the cardiovascular events in diabetic patients [20]. Contradictory data were found in literature regarding the role of vitamin $E$ in protection against cardiac disease. Hu et al. [13] found that vitamin E exerted harmful effect in young female mice, as it increased cardiomyocyte apoptosis after induction of myocardial infarction (MI) while it is protective in aged male mice.

Myocardial infarction is an ischaemia-induced cardiac muscle necrosis which results from "imbalance between coronary blood supply and myocardial demand" [32]. MI induced using isoproterenol was adequately reproduced by many researchers [11]. It was used to test therapeutic or cardioprotective effect of any new medication or adjuvant natural supplementation [7]. Therefore, this study was designed to evaluate the effectiveness of L-carnitine, vitamin $\mathrm{E}$ and a combination of both in protection against isoproterenol-induced biochemical histopathological changes in rat heart.

\section{MATERIALS AND METHODS}

\section{Ethical approval}

This study was conducted after obtaining the ethical approval of the Biomedical Research Ethics Committee, Faculty of Medicine, King Abdulaziz University, Jeddah Saudi Arabia and was done at the King Fahad Medical Research Centre (KFMRC), King Abdulaziz University.

\section{Drug}

Isoproterenol hydrochloride was obtained from Sigma chemical company, St. Louis, MO, USA. Vitamin E and L-carnitine were obtained from GNC Store at Jeddah, Saudi Arabia.

\section{Animals}

Fifty male Westar albino rats weighing from 150 to $200 \mathrm{~g}$ and were purchased from the experiment animal unit at the KFMRC. They were kept in large cages at $25^{\circ} \mathrm{C}$ with a 12 -h dark/light cycle with free mobility and allowed free access to water and standard rat diet ad libitum. They were left to acclimatise for 2 weeks in their cages before start the experiment.

\section{Experimental protocols}

Rats were randomly assigned to five groups $(n=10)$.

- Group I - served as a control group. Rats of this group were injected subcutaneously with $2 \mathrm{~mL}$ of saline daily for 4 weeks;

- Group II - in which rats were subcutaneously injected with isoproterenol $(100 \mathrm{mg} / \mathrm{kg}$ body weight (BW) in $2 \mathrm{~mL}$ of saline daily for 3 successive days according to Panda and Kar [23];

- Group III - in which rats were subcutaneously injected with isoproterenol with same dose and duration plus vitamin $\mathrm{E}(100 \mathrm{lU} / \mathrm{kg} \mathrm{BW})$ intraperitoneal injection daily for 4 weeks according to Gayathri et al. [8];

- Group IV - in which rats were subcutaneously injected with isoproterenol plus intraperitoneal daily injection of L-carnitine (100 mg/kg BW) for 4 weeks according to Wong et al. [33];

- Group V - in which rats were subcutaneously injected with isoproterenol plus vitamin $\mathrm{E}$ and L-carnitine at the same dose for 4 weeks.

\section{Biochemical assessment}

At the end of the experiment, blood sample was collected from the retro-orbital vein under after thiopental anaesthesia. Blood was centrifuged at $4000 \mathrm{~g}$ at $4^{\circ} \mathrm{C}$ for $15 \mathrm{~min}$ and the serum was stored at $-80^{\circ} \mathrm{C}$ till the time of the biochemical assessment of serum aspartate transaminase (AST), lactate dehydrogenase $(\mathrm{LDH})$, and creatine kinase (CK-MB) by colorimetric method using commercial KIT from Biomedical [30]. Rats were then sacrificed with cervical dislocation, the chest was open and the heart was immediately dissected out.

Part of the heart wall was homogenised in 9 volumes of ice-cold saline and centrifuged at $8000 \mathrm{~g}$ at $4^{\circ} \mathrm{C}$ for $20 \mathrm{~min}$. Malondialdehyde (MDA) level was assessed in the homogenate as was described according to Moran [21]. Superoxide dismutase (SOD) activity 
was assessed in the homogenate by the nitro blue tetrazolium reduction method according to Habig et al. [12]. Glutathione peroxidase (GSPxase) was assessed as described by Pagila and Valentine [22] and glutathione reductase (GSRase) was estimated according to Deore et al. [6]. Levels of inflammatory cytokines interleukin 6 (IL-6), tumour necrosis factor alpha (TNF- $\alpha$ ) were measured by ELISA kits from BIORAD (England).

\section{Histopathological assessment}

The dissected heart was washed with saline solution, cut transversely and longitudinally and fixed in $10 \%$ buffered neutral formalin solution, and processed for obtaining paraffin blocks. These blocks were sectioned serially at 4-6 $\mu$ and routinely stained with haematoxylin and eosin (H\&E) [3].

Specimens measured about $2 \times 2 \mathrm{~mm}$ from left ventricle were fixed in $3 \%$ glutaraldehyde in phosphate buffer at $\mathrm{pH} 7.4$ for $24 \mathrm{~h}$, and post-fixed in $1 \%$ osmic acid for $1 \mathrm{~h}$ and further processed to be examined with transmission electron microscopy (TEM) (JEM-100 Cx11; Jeol) at Assuit University, Assuit, Egypt. In order to determine the orientation of the ultrathin sections, semithin ones with thickness $1 \mathrm{~mm}$ were prepared and stained using toluidine blue to be examined by the light microscope. The ultrathin sections (prepared at the thickness of $500-800 \AA$ ) were stained with uranyl acetate and lead citrate.

\section{RESULTS}

\section{Biochemical findings}

In this study, rats that received isoproterenol (ISO) showed a significant increase in the serum level of cardiac enzymes including AST, LDH and CK-MB compared to the control rats. Treating rats with vitamin $\mathrm{E}$, L-carnitine or both along with ISO resulted in a significant reduction in these enzymes levels compared to the untreated rats. However, the level of these enzymes did not reach that of the control group. The combined effect of both vitamin $\mathrm{E}$ and L-carnitine was more significant on the level of CK-MB compared with LDH and AST (Fig. 1A). The level of MDA, a marker of oxidative stress, was assessed in the cardiac tissue homogenate. It was observed that ISO significantly elevated MDA level in the cardiac homogenate compared to the control group. Administration of vitamin $E$, L-carnitine or both along with ISO significantly decreased in MDA in the cardiac tissue compared to the untreated group (Fig. 1B).
The levels of SOD, GSPxase and GSRase, the cardiac antioxidant enzymes, were assessed in the homogenate of the cardiac tissue. It was observed that administration of ISO induced a significant increase in the level of SOD and a significant decrease in the level of GSPxase and GSRase compared with the control group. Administration of vitamin E, L-carnitine or both along with ISO significantly reduce the level of SOD and significantly increased the levels of GSPxase and GSRase when compared with the untreated group. Although none of these antioxidants reached the level observed in the control group, the combined effect of both vitamin E and L-carnitine was more significant on the level of GSRase compared with other enzymes (Fig. 1C). When it came to the levels of the inflammatory cytokines, it was found that the levels of IL- 6 and TNF- $\alpha$ showed a significant increase in the cardiac tissue after administration of ISO while their levels were significantly reduced in rats receiving both vitamin $\mathrm{E}$ and L-carnitine, compared with the untreated rats (Fig. 1D, E).

\section{Histopathological results}

Light microscopic findings. The light microscopic inspection of the left ventricle of the control rat revealed intact cylindrical cardiac muscle fibres, with acidophilic cytoplasm and ill-defined transverse striations. The nuclei of the cardiac fibres were vesicular, oval or rounded and central in position. Connective tissue between the fibres was scanty and contained thin wall blood capillaries and few fibroblasts identified by their flat nuclei. The blood vessels, which were branches of the coronary vessels between the cardiac muscle fibres, had intact wall with normal structure and perivascular tissue (Fig. 2A, B).

Isoproterenol administration induced histological alterations including atrophy of some cardiac fibres and degeneration of some fibres, which was recognised by the dark cytoplasm and small pyknotic nuclei. Some capillaries appeared congested or ruptured, resulting in haemorrhagic foci between the fibres. The blood vessels between the fibres appeared damaged with perivascular inflammatory cells around them (Fig. 2C, D). Sections in the heart wall of groups treated with vitamin E, L-carnitine or their combination showed that most of the cardiac muscle were intact while few fibres were degenerated and the blood vessels appeared normal similar to control (Fig. 2E, J).

Semithin sections prepared from the same samples confirmed what was observed by routine histo- 


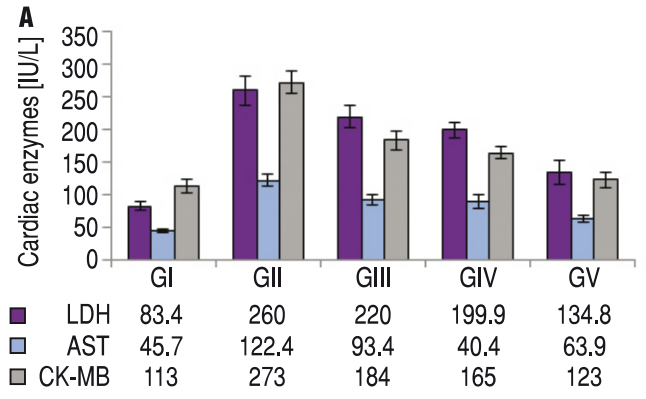

B
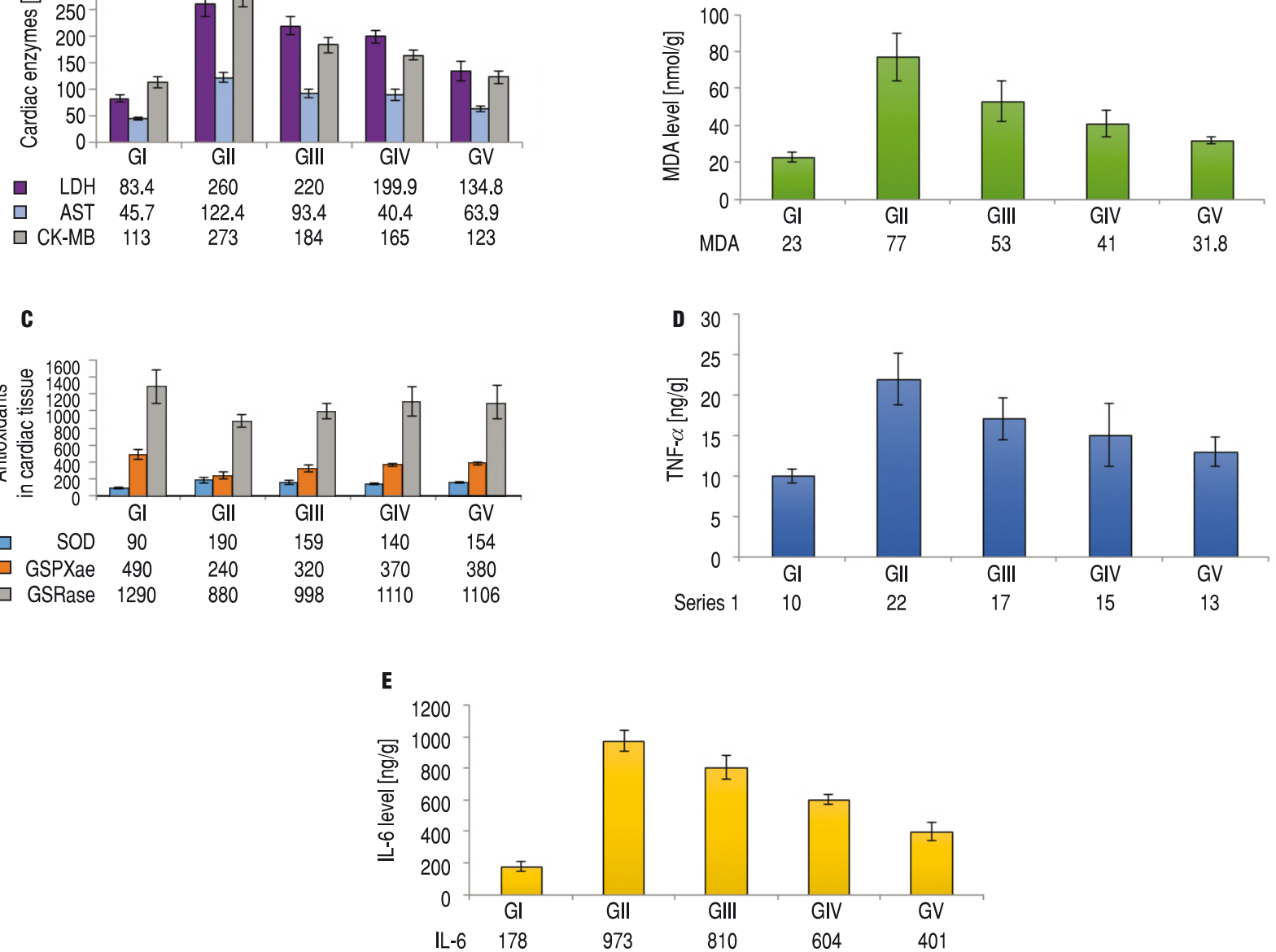

Figure 1. Changes in cardiac enzyme (lactate dehydrogenase [LDH], aspartate transaminase [AST], creatine kinase [CK-MB]) (A), malondialdehyde (MDA) (B), antioxidant (C), tumour necrosis factor alpha (TNF- $\alpha$ ) and interleukin 6 (IL-6) levels (D) in the studied groups; $\mathrm{GI}$ - control group; GII — isoproterenol group; GIII — isoproterenol + vitamin E group, GIV — isoproterenol + L-carnitine group; GV — isoproterenol + vitamin E + L-carnitine group; GSPxase — glutathione peroxidase; GSRase — glutathione reductase; SOD — superoxide dismutase.

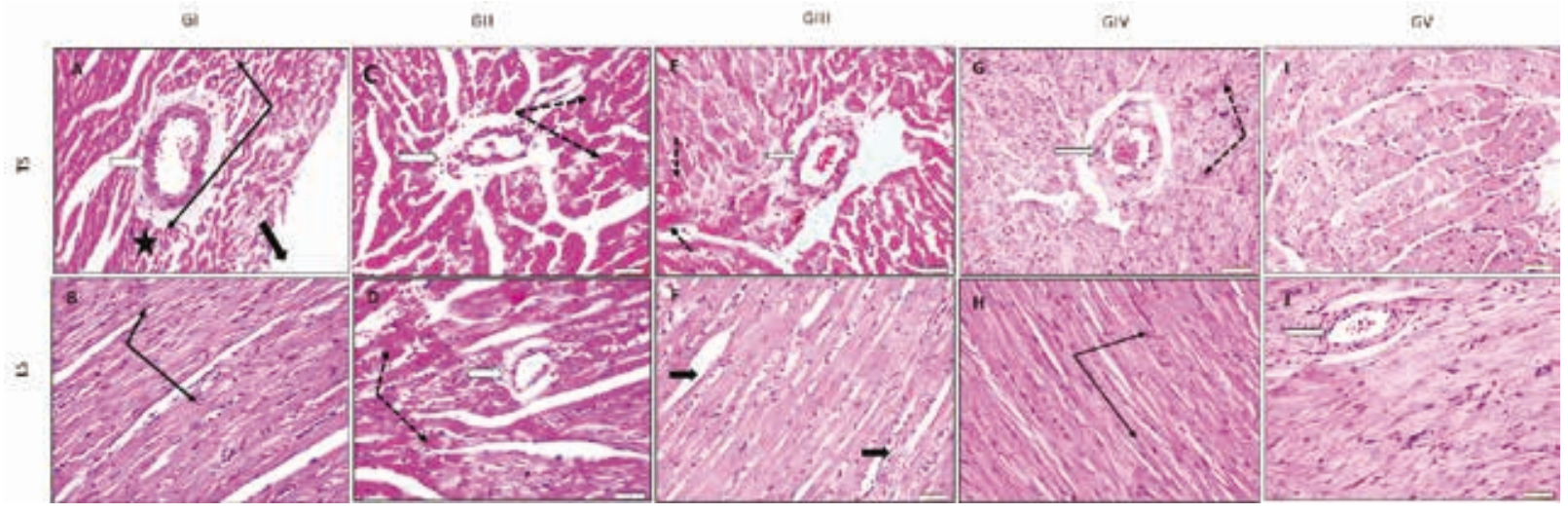

Figure 2. Transverse (A) and longitudinal (B) sections in control rat heart (GI) showed intact cardiac muscle fibres (black arrow) and intact blood vessels (white arrow). Sections in heart of GII rats (C, D) show atrophy and decreased size of cardiac fibres (thick arrows) with some haemorrhages in between them (asterisk). Some muscle fibres appear degenerated with dark cytoplasm and small pyknotic nuclei (interrupted arrow). The blood vessel (white arrow) appears damaged with perivascular inflammatory cells around. Sections in the heart of Glll rats $(\mathbf{E}, \mathbf{F})$ and those of GIV rats $(\mathbf{G}, \mathbf{H})$ showing few degenerated muscle fibres (interrupted arrows) and the blood vessels appear intact. Sections in the heart of GV rats (I, J) showing almost intact muscle fibres and blood vessels (H\&E, $\times 400)$; GI — control group; GII — isoproterenol group; GIII — isoproterenol + vitamin E group, GIV — isoproterenol + L-carnitine group; GV — isoproterenol + vitamin E + L-carnitine group; LS — longitudinal section; TS — transverse section. 


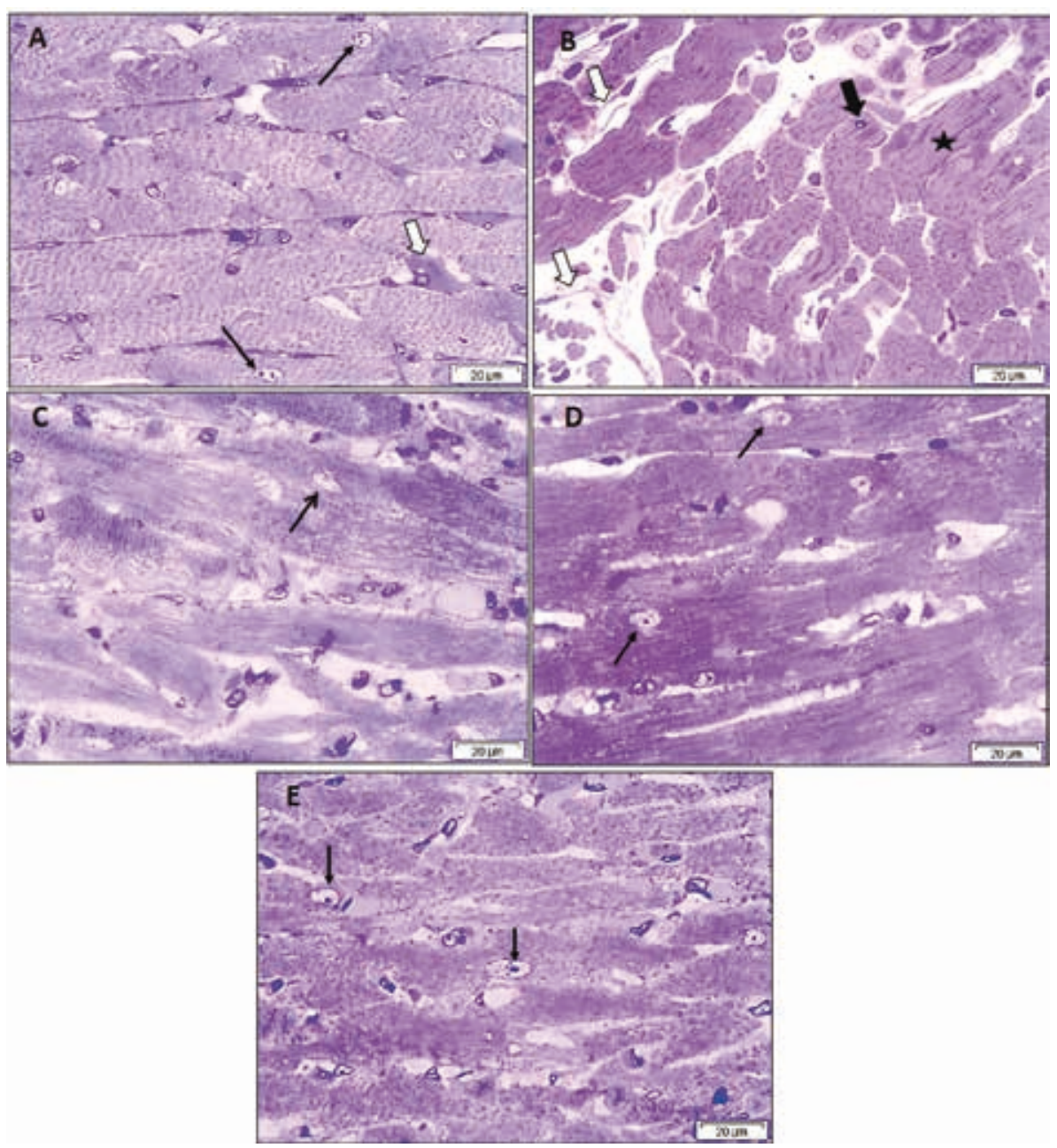

Figure 3. Semithin sections in the heart of $\mathrm{GI}$ (A) showing intact cardiac muscle fibres with vesicular central nuclei (arrows) and intact striation. Note, the presence of thin-walled capillaries (white arrow). Section in the heart of GII (B) showing reduced diameter of muscle fibres; some of these fibres have dark small nuclei (thick arrow) and some lost their striations (asterisk). Note that most of blood capillaries are dilated and congested (white arrow). Sections in hearts of GIII (C), GIV (D) and GV (E) show that most of muscle fibres are intact with vesicular nuclei (arrow). Striations are preserved in most of the muscle fibres while few lost them (toluidine blue stain, $\times 600$ ); $\mathrm{GI}$ - control group; GII — isoproterenol group; GIII — isoproterenol + vitamin E group, GIV — isoproterenol + L-carnitine group; GV — isoproterenol + vitamin E + L-carnitine group.

logical stains. The cardiac muscle fibres of the control rats appeared intact with preserved striation and intact capillaries in between. Evident focal damage of cardiac fibres was observed in rats receiving ISO as some fibres lost their striation compared to the control. Most of blood capillaries were dilated and congested. On the other hand, most of the cardiac muscle fibres in the hearts of the treated groups showed preserved striations while few fibres had lost them. The rats receiving both vitamin $E$ and L-carnitine showed the best effect on preserving the structure of the cardiac muscle (Fig. 3).

Electron microscopic findings. When the ultrastructure of the cardiac muscle fibres was assessed using TEM, it was noticed that the control rats showed euchromatic nuclei, intact, well-defined sarcomeres and intact mitochondria with normal size and shape. On the other hand, cardiac fibres of rats receiving ISO appeared atrophied with occasional ill-defined sarcomeres, smaller mitochondria with disrupted cristae. Although the cardiac fibres of rats treated with vitamin $\mathrm{E}$, L-carnitine or a combination of both possessed preserved sarcomeres, the mitochondria still appeared smaller in size and less frequently seen in the fibres compared to the control rats (Figs. 4, 5).

\section{DISCUSSION}

"Myocardial infarction is a condition that results from the interruption of coronary blood supply needed to satisfy myocardial demand, leading to 

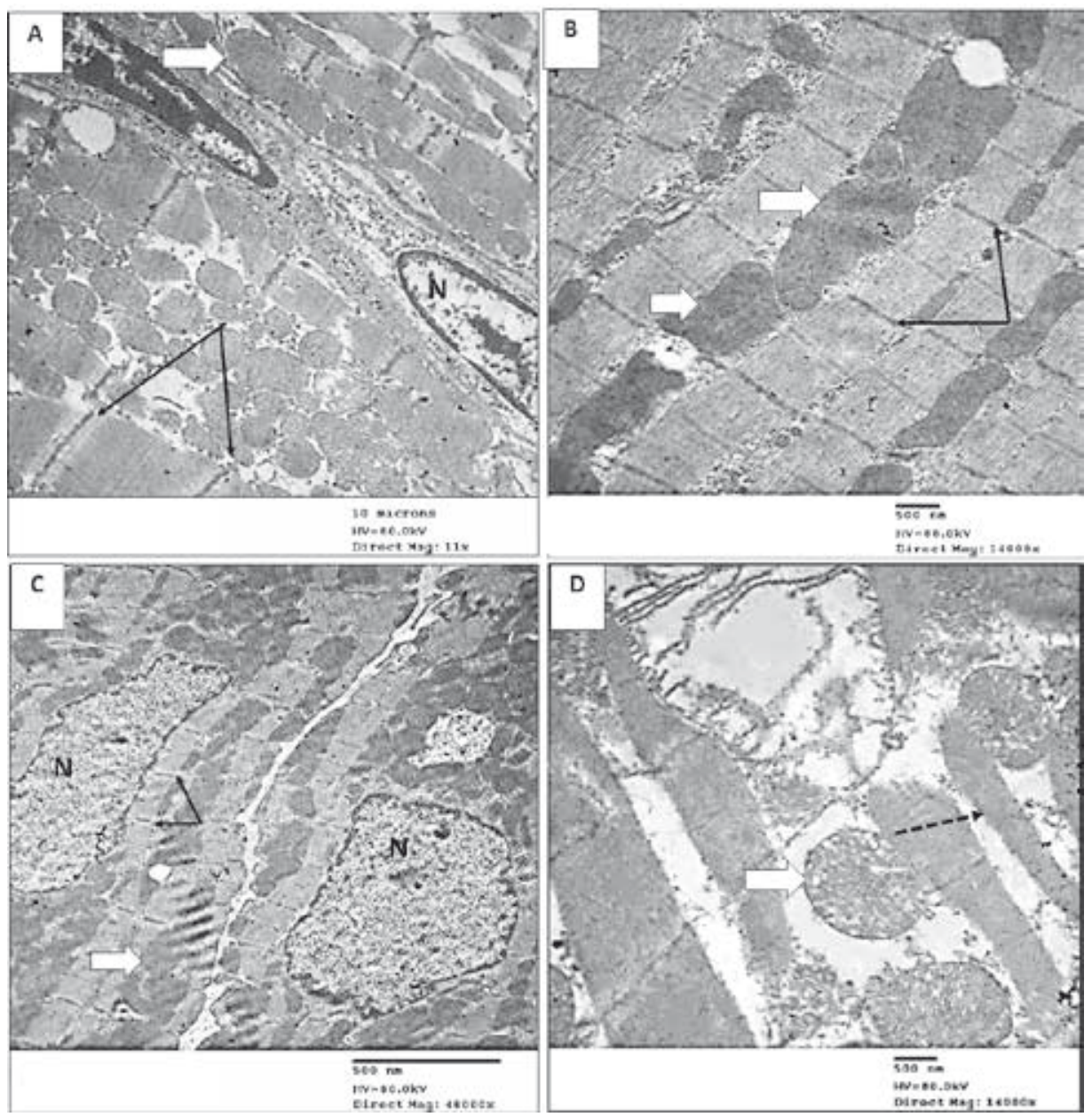

Figure 4. Electron microscope micrograph of rat cardiac muscle from the left ventricle of control group (GI) $(\mathbf{A}, \mathbf{B})$ showing normal structure of the cardiac fibres with open face nuclei (N), well defined sarcomeres (black arrows) and normal size and site of mitochondria (thick white arrows). Cardiac muscle of isoproterenol-treated group (GII) (C, D) showing reduced diameter (atrophy) of cardiac muscles (interrupted arrow) with occasional ill-defined sarcomeres (arrows). Mitochondria appear smaller in size with disrupted cristae (thick white arrows).

oxygen and nutrient deprivation of the heart, eventually destroying cardiac tissues" [5]. "Isoproterenol, a synthetic catecholamine and adrenergic agonist that is documented to produce in large dose due to generation of highly cytotoxic free radicals through its auto-oxidation". These free radicals initiate lipid peroxidation and induce irreversible damage of the myocardial membrane [9]. This study was planned to assess the possible protective effect of vitamin $E$, L-carnitine, and a combination of both on ISO-induced changes in rat cardiac muscle.

The diagnostic cardiac enzymes CK-MB, LDH and AST are considered sensitive markers that are used to assess the severity of MI [32]. In this study, ISO significantly increased the levels of diagnostic cardiac enzymes (LDH, AST and CK-MB) in the serum and MDA level in the cardiac tissue compared with the control rats. In addition, it significantly increased SOD activity and significantly decreased GSPxase and GSRase activity indicating its harmful effect on the cardiac muscle. These findings were supported by those of Panda et al. [24] observed in rats after administration of ISO.

Free radicals generated by ISO stimulate lipid peroxidation of polyunsaturated fatty acid of the membrane, with subsequent loss of the structure and function of the myocardium. When the metabolic function of the myocardium is disturbed, it releases its lysosomal enzymes into the blood. Therefore, the serum levels of AST, LDH and CK-MB were found to be significantly increased [19].

Isoproterenol induced structural alterations in cardiac muscle. Among these alternations were the appearance of some muscle fibres with dark cytoplasm and small pyknotic nuclei, indicating apoptosis and 

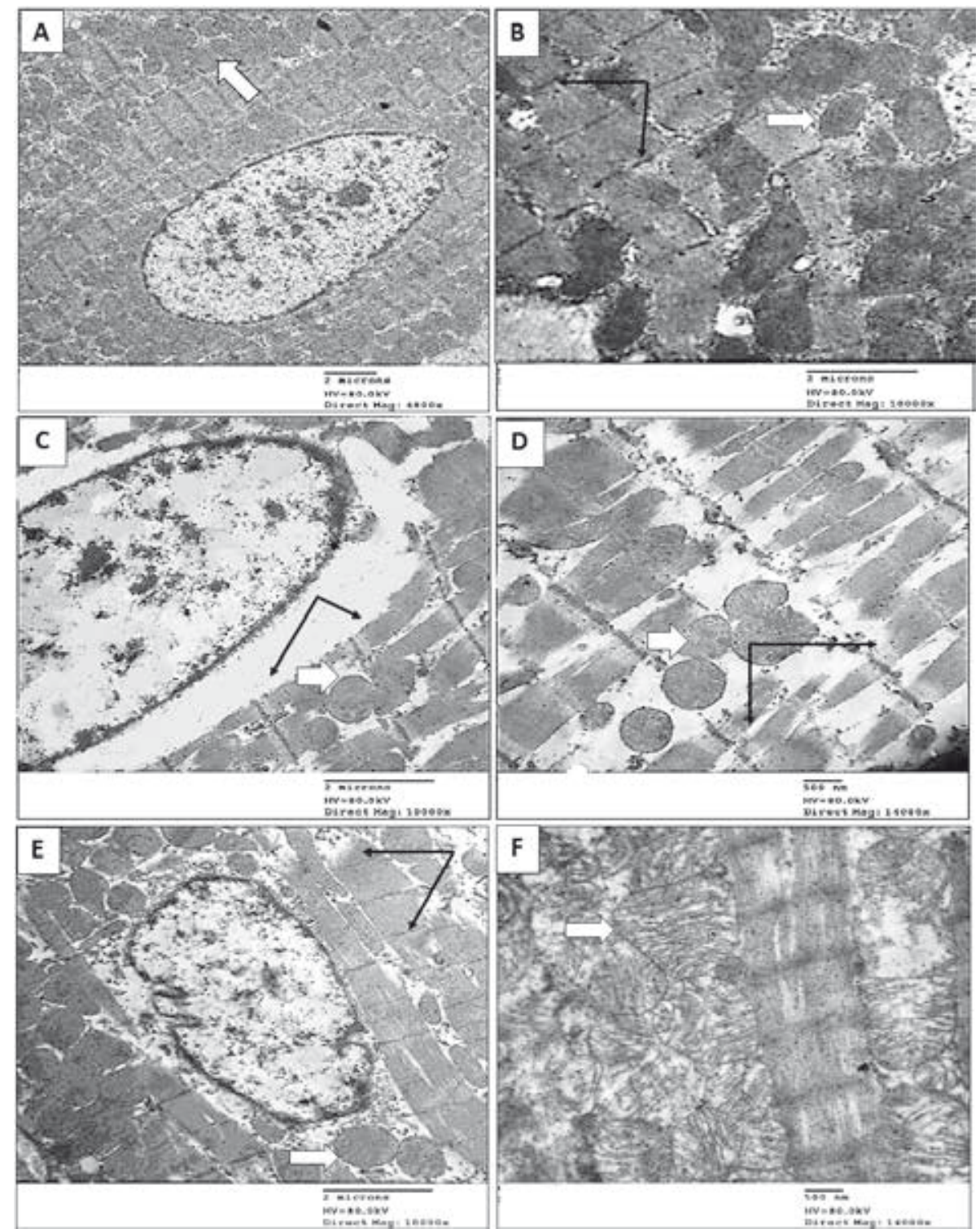

Figure 5. Electron microscope micrograph of rat cardiac muscle from the left ventricle of isoproterenol + vitamin E group (GIII) (A, B), isoproterenol + L-carnitine group (GIV) (C, D) and isoproterenol + vitamin E + L-carnitine group (GV) (E, F) showing increased diameter of most of the cardiac fibres, defined sarcomeres (black arrows) while the mitochondria (thick white arrows) still have small size and fewer number compared to the control group.

degeneration of these fibres. These finding was supported by those previous reported by Shukla et al. [28] who stated that ISO initiates apoptosis evidenced by up-regulation of TUNEL and Bax expression and down regulation of Bcl-2 expression. Atrophy of cardiac muscle fibres as well as haemorrhage and congestion of some blood vessel were observed following ISO administration in this study. These finding were in agreement with what was reported by Panda et al. [24] following administration of ISO. Gayathri et al. [8] reported that ISO administration was associ- ated with vascular changes, oedema and leucocyte infiltration. Marked focal myonecrosis where among the histopathological changes induced by ISO in rats as described by Shukla et al. [28]. In this study, ISO administration affected also the sarcomere, the functional unit of muscle fibre, and mitochondria, which are the powerhouses of the fibre. Damage to membranous organelles, such as mitochondria, is attributed to lipid peroxidation induced by ISO [10]. Tappia et al. [29] reported that "oxidative stress increases cAMP levels by exhausting ATP, depresses 
sarcolemmal $\mathrm{Ca}^{2+}$ transport resulting in intracellular calcium overload, leading to ventricular dysfunction and contractile failure in rat heart".

In the present study, administration of L-carnitine was found to protect rat heart from ISO-induced changes observed in untreated group. It improved the oxidative stress induced by ISO as evidenced by the significant reduction in MDA. In addition, L-carnitine increased antioxidant capacity of the cardiac muscle evidenced by increased GSPxase and GSRase activity. In another model of $\mathrm{MI}$ induced by amethopterin, L-carnitine was found to improve the biochemical, histopathological, and immunohistochemical alterations followed amethopterin administration [31]. Previously L-carnitine administration was reported to have a cardioprotective role in cardiomyopathy and prevention of MI [14]. Lee et al. [15] found that L-carnitine supplementation increased antioxidant enzymes, improved lipid profile and decreased oxidative stress in patients with coronary artery diseases.

Vitamin $E$ in the present study was also found to ameliorate degenerative changes and other cardiac fibre alterations induced by ISO administration. This protection was observed at both light and electron microscopic level. In addition, vitamin E reduced ISO-induced oxidative stress as evidenced by reduction in MDA. Vitamin E also increased cardiac muscle antioxidant capacity evidenced by increased GSPxase and GSRase activity. The anti-inflammatory effect of vitamin $\mathrm{E}$ was evident as it significantly reduced the elevated inflammatory cytokines IL- 6 and TNF- $\alpha$ in the cardiac tissue after administration of ISO. These finding were supported by the study of Boaz et al. [4] and Lee et al. [16], who stated that vitamin E was known to be a potent antioxidant with proven effectiveness in prevention of cardiovascular diseases. It has been proposed that the cardio protective effect of L-carnitine and vitamin $\mathrm{E}$ is due to anti-inflammatory and antioxidant activities on coronary endothelial function and vasoreactivity [34]. This could explain the better effect induced by the combination of L-carnitine and vitamin $\mathrm{E}$ in protection against ISO-induced cardiac injury that was documented in this study.

Among the limitations of this study was the inability to assess changes in gene expression of the antioxidant profile in the cardiac muscle in all studied groups in order to prove the mechanism behind the augmentation of action between vitamin $\mathrm{E}$ and L-carnitine.

\section{CONCLUSIONS AND CLINICAL IMPLICATIONS}

This biochemical and histological studies demonstrated that administration of vitamin $E$ and L-carnitine ameliorated cardiac alteration induced by ISO with the superior effect of the combination of both agents. Based on these findings it is likely that administration of these two elements is recommended for patient at risk of $\mathrm{Ml}$ as they exert a cardio protective effect by stabilizing the myocardial membrane. The membrane-stabilising activity may be due to an increase in endogenous antioxidants, which may increase the myocardial antioxidant reserve and strengthen the defence mechanism(s) in the myocardium.

\section{REFERENCES}

1. Ahmed MM, Ibrahim ZS, Alkafafy M, et al. L-carnitine protects against testicular dysfunction caused by gamma irradiation in mice. Acta Histochem. 2014; 116(6): 1046-1055, doi: 10.1016/j.acthis.2014.04.010, indexed in Pubmed: 24925768.

2. Alshiek JA, Dayan L, Asleh R, et al. Anti-oxidative treatment with vitamin $\mathrm{E}$ improves peripheral vascular function in patients with diabetes mellitus and Haptoglobin 2-2 genotype: A double-blinded cross-over study. Diabetes Res Clin Pract. 2017; 131: 200-207, doi: 10.1016/j.diabres.2017.06.026, indexed in Pubmed: 28759833.

3. Bancroft JD, Gamble M. Theory and practice of histological techniques. 6th ed. Churchill Livingstone, Elsevier, China. 2008.

4. Boaz M, Smetana S, Weinstein T, et al. Secondary prevention with antioxidants of cardiovascular disease in endstage renal disease (SPACE): randomised placebo-controlled trial. Lancet. 2000; 356(9237): 1213-1218, indexed in Pubmed: 11072938.

5. De Bono DP, Boon NA. Diseases of the cardiovascular system. In: Edwards CRW, Bouchier IAS, eds. Davidson's Principles of Practice and Medicine. Hong Kong: Churchill Livingstone. 1992: 249-340.

6. Deore AB, Vinayak D. Sapakal and Naikwade NS: Antioxidant and hepatoprotective activity of Garcinia indica Linn fruit rind. J Comprehensive Pharmacy. 6.08.

7. Dhivya V, Priya LB, Chirayil HT, et al. Piperine modulates isoproterenol induced myocardial ischemia through antioxidant and anti-dyslipidemic effect in male Wistar rats. Biomed Pharmacother. 2017; 87: 705-713, doi: 10.1016/j. biopha.2017.01.002, indexed in Pubmed: 28088738.

8. Gayathri K, Jayachandran KS, Vasanthi HR, et al. Cardioprotective effect of lemon grass as evidenced by biochemical and histopathological changes in experimentally induced cardiotoxicity. Hum Exp Toxicol. 2011; 30(8): 1073-1082, doi: 10.1177/0960327110386391, indexed in Pubmed: 20952451.

9. Goyal S, Siddiqui MK, Siddiqui KM, et al. Cardioprotective effect of 'Khamira Abresham Hakim Arshad Wala' a unani formulation in isoproterenol-induced myocardial necrosis in 
rats. Exp Toxicol Pathol. 2010; 62(1): 61-74, doi: 10.1016/j. etp.2009.02.115, indexed in Pubmed: 19285846.

10. Goyal SN, Arora S, Sharma AK, et al. Preventive effect of crocin of Crocus sativus on hemodynamic, biochemical, histopathological and ultrastuctural alterations in isoproterenol-induced cardiotoxicity in rats. Phytomedicine. 2010; 17(3-4): 227-232, doi: 10.1016/j.phymed.2009.08.009, indexed in Pubmed: 19747807.

11. Goyal SN, Sharma C, Mahajan UB, et al. Protective effects of cardamom in isoproterenol-induced myocardial infarction in rats. Int J Mol Sci. 2015; 16(11): 27457-27469, doi: 10.3390/ijms161126040, indexed in Pubmed: 26593900.

12. Habig WH, Pabst MJ, Jakoby WB. Glutathione S-transferases. The first enzymatic step in mercapturic acid formation. J Biol Chem. 1974; 249(22): 7130-7139, indexed in Pubmed: 4436300.

13. Hu XX, Fu Li, Li Y, et al. The cardioprotective effect of vitamin e (alpha-tocopherol) is strongly related to age and gender in mice. PLoS One. 2015; 10(9): e0137405, doi: 10.1371/ journal.pone.0137405, indexed in Pubmed: 26331272.

14. Lango R, Smolenski RT, Narkiewicz M, et al. Influence of L-carnitine and its derivatives on myocardial metabolism and function in ischemic heart disease and during cardiopulmonary bypass. Cardiovasc Res. 2001; 51(1): 21-29, indexed in Pubmed: 11399244.

15. Lee BJ, Lin JS, Lin YC, et al. Effects of L-carnitine supplementation on lipid profiles in patients with coronary artery disease. Lipids Health Dis. 2016; 15: 107, doi: 10.1186/ s12944-016-0277-5, indexed in Pubmed: 27317162.

16. Lee IM, Cook N, Gaziano J, et al. Vitamin E in the primary prevention of cardiovascular disease and cancer. The Women's Health Study: a randomized controlled trial. JAMA. 2005; 294(1): 56-65, doi: 10.1001/jama.294.1.56.

17. Li Li, Zhu Di, Sun X. [Cellular uptake of TPS-L-carnitine synthesised as transporter-based renal targeting prodrug]. Sichuan Da Xue Xue Bao Yi Xue Ban. 2012; 43(6): 936-940, indexed in Pubmed: 23387232.

18. Liepinsh E, Makrecka-Kuka M, Kuka J, et al. Inhibition of L-carnitine biosynthesis and transport by methyl- $\gamma$-butyrobetaine decreases fatty acid oxidation and protects against myocardial infarction. Br J Pharmacol. 2015; 172(5): 1319-1332, doi: 10.1111/bph.13004, indexed in Pubmed: 25363063.

19. Panteghini M. Role and importance of biochemical markers in clinical cardiology. Eur Heart J. 2004; 25(14): 1187-1196, doi: 10.1016/j.ehj.2004.04.026.

20. Milman U, Blum S, Shapira C, et al. Vitamin E supplementation reduces cardiovascular events in a subgroup of middleaged individuals with both type 2 diabetes mellitus and the haptoglobin 2-2 genotype: a prospective double-blinded clinical trial. Arterioscler Thromb Vasc Biol. 2008; 28(2): 341-347, doi: 10.1161/ATVBAHA.107.153965, indexed in Pubmed: 18032779.

21. Moran MS. Levels of glutathione, glutathione reductase and glutathione-S-transferase activity in rat lung and liver. Biochim et Biophys. 1979; 582: 67-78.

22. Paglia DE, Valentine WN. Studies on the quantitative and qualitative characterization of erythrocyte glutathione peroxidase. J Lab Clin Med. 1967; 70(1): 158-169, indexed in Pubmed: 6066618.
23. Panda S, Kar A. Combined effects of vincristine and quercetin in reducing isoproterenol-induced cardiac necrosis in rats. Cardiovasc Toxicol. 2015; 15(4): 291-299, doi: 10.1007/ s12012-014-9291-x, indexed in Pubmed: 25537132.

24. Panda V, Kamble S, Desai Y, et al. Antioxidant and cardioprotective effects of Garcinia indica (kokoberry), an Indian super fruit in isoproterenol induced myocardial necrosis in rats. J Berry Res. 2014; 4(3): 159-174, doi: 10.3233/jbr-140078.

25. Salama A, Kasem S, Tousson E, et al. L-carnitine and vitamin E alleviate reproductive toxicity caused by triton WR 1339 in male albino rats. Toxicol Ind Health. 2015; 31(5): 467-474.

26. Schönekess B, Lopaschuk $G$. The effects of carnitine on myocardial carbohydrate metabolism. The Carnitine System. A New Therapeutical Approach to Cardiovascular Diseases, edited by De Jong JW, Ferrari R, Dordrecht, Kluwer Academic Publishers. 1995: 39-52, doi: 10.1007/978-94011-0275-9_4.

27. Shang R, Sun Z, Li H. Effective dosing of L-carnitine in the secondary prevention of cardiovascular disease: a systematic review and meta-analysis. BMC Cardiovasc Disord. 2014; 14: 88, doi: 10.1186/1471-2261-14-88, indexed in Pubmed: 25044037.

28. Shukla SK, Sharma SB, Singh UR, et al. Eugenia jambolana pretreatment prevents isoproterenol-induced myocardial damage in rats: evidence from biochemical, molecular, and histopathological studies. J Med Food. 2014; 17(2): 244-253, doi: 10.1089/jmf.2013.2795, indexed in Pubmed: 24325453.

29. Tappia PS, Hata T, Hozaima L, et al. Role of oxidative stress in catecholamine-induced changes in cardiac sarcolemmal Ca2+ transport. Arch Biochem Biophys. 2001; 387(1): 85-92, doi: 10.1006/abbi.2000.2234, indexed in Pubmed: 11368187.

30. Thygesen K, Mair J, Katus H, et al. Acute Cardiac Care. Recomendations for the use of cardiac troponin measurement in acute cardiac care. Eur Heart J. 2010; 31(18): 2197-2204, doi: 10.1093/eurheartj/ehq251, indexed in Pubmed: 20685679.

31. Tousson E, Hafez E, Zaki S, et al. The cardioprotective effects of L-carnitine on rat cardiac injury, apoptosis, and oxidative stress caused by amethopterin. Environ Sci Pollut Res Int. 2016; 23(20): 20600-20608, doi: 10.1007/s11356-0167220-1, indexed in Pubmed: 27464663.

32. Whellan D. Heart failure disease management. Implementation and outcomes. Cardiol Rev. 2005; 13(5): 231-239, doi: 10.1097/01.crd.0000135765.60824.2f.

33. Wong GK, Pehora C, Crawford MW. L-carnitine reduces susceptibility to bupivacaine-induced cardiotoxicity: an experimental study in rats. Can J Anaesth. 2017; 64(3): 270-279, doi: 10.1007/s12630-016-0797-5, indexed in Pubmed: 28032304.

34. Yüncü $M$, Bükücü N, Bayat $N$, et al. The effect of vitamin $E$ and L-carnitine against methotrexate-induced injury in rat testis. Turk J Med Sci. 2015; 45(3): 517-525, indexed in Pubmed: 26281314

35. Zambrano S, Blanca AJ, Ruiz-Armenta MV, et al. L-carnitine protects against arterial hypertension-related cardiac fibrosis through modulation of PPAR- $\gamma$ expression. Biochem Pharmacol. 2013; 85(7): 937-944, doi: 10.1016/j. bcp.2012.12.021, indexed in Pubmed: 23295156. 